\title{
Außenwirtschaft in Zeiten der Globalisierung - Determinanten, Effekte und Entwicklungen von Direktinvestitionen
}

\author{
Hans Wolfgang Brachinger
}

Online: 17. November 2009

(C) Springer 2009

Im Rahmen der Reihe „,Dialog von Wissenschaft und amtlicher Statistik“ veranstaltete der Rat für Sozial- und Wirtschaftsdaten (RatSWD) zusammen mit der Deutschen Bundesbank und dem Statistischen Bundesamt am 22. und 23. April 2008 in Wiesbaden eine Konferenz zum Thema „Außenwirtschaft in Zeiten der Globalisierung - Möglichkeiten und Grenzen der statistischen Messung“. In den Konferenzbeiträgen wurden neuere Entwicklungen bei der Messung wichtiger Aspekte der Internationalisierung der Wirtschaft diskutiert, neu verfügbare Datenbestände vorgestellt und Ergebnisse von empirischen Untersuchungen präsentiert.

Ein erster Teil ausgewählter Tagungsbeiträge wurde in Heft 2 dieses Jahrgangs des Wirtschafts- und Sozialstatistischen Archivs (WiSoStA) veröffentlicht. In diesen Beiträgen standen Fragen der statistischen Erfassung der Globalisierung im Vordergrund. Diese Problematik wurde aus der Sicht von Datenproduzenten und aus der von Datennutzern abgehandelt. In diesem Heft werden - in überarbeiteter Form, in die Anregungen aus der Diskussion und Hinweise der Gutachter sowie des Herausgebers der Zeitschrift eingeflossen sind - vier weitere Konferenzbeiträge publiziert. Diese Beiträge beschäftigen sich mit dem Thema „Direktinvestitionen“.

Sebastian Hügelschäffer, Daniel Kromer und Alexander Lipponer (Hügelschäffer et al. 2009) stellen in ihrem Aufsatz ,Die deutsche Statistik über Direktinvestitionen: Neuerungen und wissenschaftliche Nutzung" neue Entwicklungen bei der deutschen Statistik über Direktinvestitionen vor und geben einen Einblick in die vielfältige wissenschaftliche Nutzung dieser Daten. Sie weisen darauf hin, dass im Zuge der wachsenden grenzüberschreitenden Verflechtung der Volkswirtschaften die Bedeutung der Direktinvestitionsstatistik zunimmt, gleichzeitig aber die Erstellung konsistenter Statistiken und die Durchführung aussagekräftiger wissenschaftlicher Studien

H. W. Brachinger $(\varpi)$

Department of Quantitative Economics, University of Fribourg,

Boulevard de Pérolles 90, 1700 Fribourg, Switzerland

E-Mail: hanswolfgang.brachinger@unifr.ch 
durch die wachsende Komplexität internationaler Konzernstrukturen erschwert wird. Dieser Artikel beschreibt zunächst Neuerungen, die es erlauben, ein differenzierteres Bild der Direktinvestitionsaktivitäten zu zeichnen. Darüber hinaus wird darauf eingegangen, wie die bei der Bundesbank verfügbaren Direktinvestitionsdaten für Forschungszwecke genutzt werden können und welche wesentlichen Ergebnisse sich aus exemplarisch ausgewählten Forschungsarbeiten ergeben haben.

Mit der Statistik über Aktivitäten ausländisch beherrschter Unternehmen in Deutschland (Inward-FATS ${ }^{1}$ ) und Tochterunternehmen deutscher Investoren im Ausland (Outward-FATS) soll künftig ein wichtiger Aspekt der Verflechtung der deutschen Wirtschaft mit dem Ausland dargestellt werden. In dem Aufsatz „Die künftige Statistik über ausländisch beherrschte Unternehmen in Deutschland (Inward FATS) und Tochterunternehmen deutscher Investoren im Ausland (Outward FATS)“ informieren Peter Schmidt, Bernd Waldmüller, Jörg Feuerhake und Beatrix StejskalPassler (Schmidt et al. 2009) über diese neue Statistik, die insbesondere Struktur und wirtschaftliche Aktivitäten solcher Unternehmen erfassen soll. In diesem Aufsatz wird das Konzept dieser Statistik erstmals vorgestellt. Es wird gezeigt, aus welchen Datenquellen sie gespeist und wie sie berechnet wird. Schließlich werden erste vorläufige Ergebnisse vorgestellt und ein Ausblick auf die künftige Ergebnisdarstellung sowie die weitere Entwicklung dieser Statistik gegeben.

Im Beitrag von Christian Arndt und Anselm Mattes (Arndt u. Mattes 2009) wird anhand von Daten des IAB-Betriebspanels empirisch untersucht, welche betrieblichen Faktoren für Direktinvestitionen ausschlaggebend sind und welche Auswirkungen sie zeitigen. In ihrem Aufsatz ,Determinanten und Effekte von Direktinvestitionen im Ausland am Beispiel baden-württembergischer Betriebe" wird im Einklang mit neueren mikroökonomischen Theorien heterogener Unternehmen auf Grundlage der repräsentativen Mikrodaten des IAB-Betriebspanels Baden-Württemberg gezeigt, dass zwar nur ein sehr kleiner Teil aller Betriebe überhaupt im Ausland investiert, jedoch nahezu ein Achtel aller sozialversicherungspflichtig Beschäftigten in diesen Betrieben tätig ist. Es sind vor allem produktive und große Betriebe, die den Schritt ins Ausland wagen. Regressionsansätze zur Erklärung der Arbeitsnachfrage zeigen, dass Direktinvestitionen im Ausland keine negativen Auswirkungen auf das betriebliche Beschäftigungsniveau haben. Es wird deutlich, dass horizontale Direktinvestitionen signifikant positive Beschäftigungswirkungen haben, vertikale dagegen keine negativen.

Gegenstand der Arbeit von Vivien Procher (Procher 2009) sind Standortentscheidungen von französischen Firmen, die erstmals als Direktinvestoren ins Ausland gehen. In ihrer Arbeit „FDI location choices: Evidence from French first-time movers“ untersucht die Verfasserin die Standortdeterminanten von französischen Erstinvestoren in Europa, Nordafrika und Nordamerika. Grundlage ihrer Untersuchungen ist ein Datensatz, der 356 Standortentscheidungen von französischen Firmen beinhaltet, die diese im Jahr 2004 in 27 Ländern getroffen haben. Marktnachfrage und kulturelle Nähe zu Frankreich haben einen großen Einfluss auf die Standortstrategie. Auslandstöchter werden vornehmlich in bestimmten Ballungszentren angesiedelt. Insbesondere die hohe Korrelation zwischen den von französischen Erstinvestoren

${ }^{1}$ Foreign AffiliaTes Statistics 
gewählten Standorten und bereits existierenden französischen Unternehmensstandorten im Ausland verdeutlicht eine gewisse Affinität zur Frankophonie. Zusätzlich werden französische Investoren oftmals auch von Firmen-Clustern anderer Nationen angezogen.

Der Prozess der Globalisierung stellt an die Datenproduzenten neue spannende Herausforderungen: Bestehende Statistiken decken die gestiegenen Informationsbedürfnisse nicht mehr ab und müssen adaptiert werden, neue statistische Daten müssen bereitgestellt werden. Die damit verbundenen Probleme werden - insbesondere auch in Deutschland - für lange Zeit im Zentrum wissenschaftlicher und amtsstatistischer Debatten stehen. Der Dialog zwischen Datenproduzenten und Datennutzern muss intensiv fortgesetzt werden. Die in den Heften 2 und 3 des Wirtschafts- und Sozialstatistischen Archivs (WiSoStA) publizierten Beiträge geben einen Überblick über den aktuellen Stand der Dinge. Mögen sie zu weiteren Aktivitäten sowohl auf Seiten der Datenproduzenten als auch auf Seiten der Wissenschaft anregen!

Der Herausgeber dankt allen Autoren für ihre Bereitschaft dem Wirtschafts- und Sozialstatistischen Archivs (WiSoStA) ihre Konferenzbeiträge zur Publikation zur Verfügung zu stellen. Sein Dank gilt insbesondere der Geduld, mit der sie den Kommentaren und Anregungen der Gutachter und des Herausgebers der Zeitschrift begegneten. Besonderer Dank gilt den Herren Hans-Peter Glaab, Joachim Wagner und Markus Zwick, die sich bei den Heften 2 und 3 des WiSoStA als Gastherausgeber engagiert haben.

\section{Hans Wolfgang Brachinger}

Herausgeber

\section{Literatur}

Arndt C, Mattes A (2009) Determinanten und Effekte von Direktinvestitionen im Ausland am Beispiel baden-württembergischer Betriebe. Wirt Sozialstat Archiv 3(3):187-208

Hügelschäffer S, Kromer D, Lipponer A (2009) Die deutsche Statistik über Direktinvestitionen: Neuerungen und wissenschaftliche Nutzung. Wirt Sozialstat Archiv 3(3):157-168

Procher V (2009) FDI location choices: Evidence from French first-time movers. Wirt Sozialstat Archiv 3(3):209-220

Schmidt P, Waldmüller B, Feuerhake J, Stejskal-Passler B (2009) Die künftige Statistik über ausländisch beherrschte Unternehmen in Deutschland (Inward FATS) und Tochterunternehmen deutscher Investoren im Ausland (Outward FATS). Wirt Sozialstat Archiv 3(3):169-186 